\title{
-Original-
}

\section{Changes in Plasma Endothelin-1 After Elective Cesarean Section in Women with Preeclampsia and the Relationship to Thrombocytopenia}

\author{
Hirobumi Asakura, Akihito Nakai and Toshiyuki Takeshita \\ Department of Obstetrics and Gynecology, Nippon Medical School Second Hospital
}

\begin{abstract}
Objective: The aim of this study was to clarify the time course of plasma endothelin-1 levels and platelet counts after elective cesarean section in women with preeclampsia, and to investigate the relationship between them postoperatively.

Methods: We studied 20 patients with preeclampsia and 25 healthy pregnant women without preeclampsia who underwent cesarean section and 20 women without preeclampsia who had vaginal deliveries. The plasma endothelin-1 and platelet counts were measured serially after cesarean section.

Results: 1) In patients with preeclampsia, plasma endothelin-1 levels peaked on postoperative day $0(\mathrm{p}<0.05)$, and remained high, then fell to the preoperative level from day 5 . The concentration was lower in healthy pregnant women undergoing cesarean section than in patients with preeclampsia before surgery, but showed a gradual significant increase from postoperative day 0 to postoperative day $3(\mathrm{p}<0.05)$ before falling. The women who underwent vaginal delivery showed a peak level of endtothelin-1 on the day of delivery but this fell rapidly between day 3. 2) Women with preeclampsia showed a negative correlation between plasma endothelin-1 levels and platelet counts after cesarean section $(r=0.46, p<0.01)$, while women without preeclampsia undergoing cesarean and vaginal deliveries did not. Conclusion: Endothelin-1 production is stimulated after cesarean section, which is paralleled with postpartal thrombocytopenia only in patients with preeclampsia.
\end{abstract}

(J Nippon Med Sch 2003; 70: 480-489)

Key words: endothelin-1, preeclampsia, cesarean section, platelet

\section{Introduction}

Plasma concentrations of endothelin-1 (ET-1) increase postoperatively due to surgical stress ${ }^{1-3}$. Hypoxia, ischemia, and thrombin formation occur in damaged tissue at wound sites, leading to the induction of a variety of cytokines, resulting in production of ET-14. Catecholamines induced by surgical stress may be a stimulator of ET-1 production. Postoperative ET-1 plays an important role in the process of wound healing, including cell migration, cell proliferation, and angiogenesis in damaged tissue ${ }^{5-8}$. Nelson et $\mathrm{al}^{2}$ argued that postoperative enhancement of ET-1 production may be linked to various postoperative complications, but there have been no studies

Correspondence to Hirobumi Asakura, Department of Obstetrics and Gynecology, Nippon Medical School Second Hospital, 1-396 Kosugi-cho, Nakahara-ku, Kawasaki City, Kanagawa 211-8533, Japan

E-mail: morgen@nms.ac.jp

Journal Website (http://www.nms.ac.jp/jnms/) 
concerning ET-1 production after cesarean sections.

The plasma level of ET-1 is already high in pregnant women with preeclampsia, as well as those with HELLP syndrome ${ }^{9-11}$. ET-1 may activate and consume platelets in blood vessels, accelerating a reduction in the platelet count ${ }^{3,12-14}$. If ET-1 production increases further after cesarean section performed for pregnant women with preeclampsia, ET1 could affect platelet functions. Actually, women with preeclampsia frequently show transient thrombocytopenia in the early postoperative days ${ }^{15,16}$. We therefore examined the time course of ET-1 levels and platelet counts after cesarean section in pregnant women with preeclampsia, in which ET-1 production was already enhanced, and studied the relationship between ET-1 levels and platelet counts.

\section{Patients and Methods}

We studied pregnant women under obstetric care at Nippon Medical School. The Nippon Medical School Ethics Committee approved the experimental protocol. Informed consent was obtained for blood collection by the participating pregnant women. Subjects were 20 pregnant women with preeclampsia and 25 healthy women without preeclampsia. Delivery was by elective cesarean section in both groups. Twenty healthy pregnant women who had vaginal deliveries served as a control. The obstetric profiles of the patients are shown in Table 1. The three groups were matched for age and parity. Twelve $(60$ $\%$ ) of the patients with preeclampsia had severe preeclampsia. Preeclampsia was defined as a systolic blood pressure of $140 \mathrm{mmHg}$ or a diastolic blood pressure of $90 \mathrm{mmHg}$, and proteinuria of $300 \mathrm{mg} / 24$ hrs, or a dipstick reading of ++ for 4 hours or longer. The preeclampsia was considered severe if any one of the following signs were present: persistent elevations in blood pressure of $\geqq 160 / 100$ $\mathrm{mmHg}$, proteinuria of $\geqq 5 \mathrm{~g} / 24 \mathrm{hrs}^{17}$. Blood pressure readings were taken at least twice every 6 hours and the diastolic pressure was the point at which the Korotkoff sound disappeared. The patients with severe preeclampsia met all of the criteria for hypertension.

The indications in the 25 pregnant women without preeclampsia for elective cesarean section included previous histories of cesarean section $(n=7)$, abnormal fetal presentation $(n=7)$, Cephalo Pelvic Disproportion $(\mathrm{CPD}) \quad(\mathrm{n}=4)$, placenta previa $(n=3)$, pregnancy after myomectomy $(\mathrm{n}=3)$ and pregnancy complicated with myom $(n=1)$.

All of the patients had single pregnancies and none had chronic hypertensive or renal diseases. None had a history of hematological or liver disease. None showed any symptoms or laboratory findings indicating HELLP syndrome before cesarean section.

Blood samples comprised venous blood taken with the patient supine. Blood was collected at the following five time points: 1) between 12 and 24 hours before cesarean section; 2 ) within $6 \sim 12$ hours after surgery (postcesarean Day 0), 3) 42 48 hours after surgery (postcesarean Day 1), 4) 88 96 hours after surgery (postcesarean Day 3), and 5) from 120 144 hours after surgery (postcesarean Day 5).

For the healthy women who underwent vaginal delivery, blood samples were taken at four time points: before onset of labor, postpartal Day 0, postpartal Day 1 and postpartal Day 3.

Blood used to measure ET-1 was collected with a chilled syringe and placed in a test tube containing aprotinin $(500 \mathrm{KU} / \mathrm{ml})$ in EDTA-2Na $(1 \mathrm{mg} / \mathrm{ml})$. Immediately after collection, the blood was centrifuged at $0^{\circ} \mathrm{C}$, after which the plasma was separated and stored at $-70^{\circ} \mathrm{C}$ until assayed. ET-1 was extracted from the plasma by absorbing the ET-1 using an octadecylsilyl silica suspension, washing with distilled water, an aqueous solution of $40 \%$ methanol, and acetone, and eluting the ET-1 with an aqueous solution of $60 \%$ methanol. The elute was then dried, and the ET-1 in it was immobilized. The ET-1 was again dissolved in a radioimmunoassay (RIA) buffer, and the sample was submitted for RIA measurement. ET-1 was measured using competitive RIA with ${ }^{125} \mathrm{I}$ as the tracer $^{17}$. Reagents kits purchased from the Peninsula Laboratory (California, USA) were used. The details of the measurement method used have been described elsewhere ${ }^{18}$. The intra-assay coefficient of variation $(\mathrm{CV})$ was $9.14 \sim 11.7 \%$, and the inter assay CV was $12.2 \sim 16.6 \%$.

Blood not used for ET-1 measurement was submitted for routine testing to measure the red blood 
Table 1 Obstetrical profiles of subjects

\begin{tabular}{l|ccc}
\hline \multirow{2}{*}{} & \multicolumn{2}{|c}{ Cesarean section } & Vaginal delivery \\
\cline { 2 - 4 } & $\begin{array}{c}\text { Preeclampsia } \\
(\mathrm{n}=20)\end{array}$ & $\begin{array}{c}\text { Nonpreeclampsia } \\
(\mathrm{n}=25)\end{array}$ & $\begin{array}{c}\text { Nonpreeclampsia } \\
(\mathrm{n}=20)\end{array}$ \\
\hline Age & $30.1 \pm 2.9$ & $29.8 \pm 3.8$ & $28.2 \pm 4.1$ \\
Nullipara & $9(45.0 \%)$ & $9(36.0 \%)$ & $10(50.0 \%)$ \\
Gestational weeks at delivery & $36.7 \pm 2.5$ & $38.1 \pm 1.9 *$ & $39.0 \pm 2.0^{*}$ \\
Neonatal birth weight $(\mathrm{g})$ & $2,239 \pm 737$ & $3,200 \pm 342^{* *}$ & $3,182 \pm 294^{*}$ \\
Total amount of bleeding $(\mathrm{m} l)$ & $731 \pm 452$ & $622 \pm 408$ & $382 \pm 207^{* *}$ \\
\hline
\end{tabular}

Data are expressed as mean \pm standard deviation and $\mathrm{n}(\%)$.

* and ${ }^{* *}$ indicate $\mathrm{p}<0.05$ and $\mathrm{p}<0.01$ vs. data from preeclamptic women who underwent cesarean section.

cell (RBC), white blood cell (WBC), hematocrit, hemoglobin, platelet counts and asparate transaminase (AST) and alanine transaminase (ALT). Laboratory tests were performed with routine methods in an autoanalyzer. Platelet counts were measured by flow cytometry. The relationship with ET-1 concentration was then studied.

\section{Statistical Analysis}

The data are all expressed as the mean \pm standard deviation. Differences between the three groups were detected using a two-way ANOVA with repeated measures followed by Schaffe's F-test. The Student's $t$ test was used to compare the values within and between the women in the study groups. The regression line was determined by the least squares method. Differences were considered significant when $\mathrm{p}<0.05$.

\section{Results}

\section{Clinical profiles of the subjects (Table 1)}

The clinical profiles of the present subjects are shown in Table 1. Ages and parity were not different in the subjects. Gestational weeks at delivery of the preeclamptic women were earlier than those of the nonpreeclamptic women undergoing cesarean section and those undergoing vaginal delivery $(\mathrm{p}<$ 0.001 ). Neonatal birth weights from the preeclamptic women were significantly lower than those from the nonpreeclamptic women who underwent cesarean section and vaginal deliveries. The total amount of bleeding was greater in the women undergoing cesarean section compared with that in the nonpreeclamptic women undergoing vaginal delivery $(\mathrm{p}<0.05)$

Five patients with preeclampsis went on to develop HELLP syndrome postpartum. The diagnosis of HELLP syndrome was made on Day 0 after cesarean section in two cases, and on postoperative Day 1 in three cases. The results were not analyzed statistically because of the small number of subjects.

\section{ET-1 levels after cesarean section (Table 2,} Fig. 1)

(1) Cesarean section.

In the women with preeclampsia, the plasma ET-1 concentration rose from $2.31 \pm 0.75 \mathrm{pg} / \mathrm{ml}$ before cesarean section to $2.92 \pm 0.82 \mathrm{pg} / \mathrm{ml}$ on Day $0(\mathrm{p}<$ 0.05). The concentrations then tended to fall on Day 1 and Day 3, but the decrease did not reach a significant level. The level decreased significantly by Day $5(\mathrm{p}<0.01)$.

The changes in ET-1 in the pregnant women with preeclampsia were significantly different from those in the pregnant women without preeclampsia who underwent cesarean section $(\mathrm{p}<0.05)$. After cesarean section, the plasma ET-1 concentration in the non-preeclamptic women rose from $1.79 \pm 0.49 \mathrm{pg} / \mathrm{ml}$ before surgery to peak at $2.53 \pm 0.84 \mathrm{pg} / \mathrm{m} l$ on Day 3 $(\mathrm{p}<0.05)$ and then recovered to the preoperative level Day $5(\mathrm{p}<0.05)$.

(2) Vaginal delivery.

ET-1 levels significantly increased on the day of delivery $(\mathrm{p}<0.01)$. However, three days after delivery the ET-1 concentration fell to the level before delivery $(p<0.05)$. A significant difference was 
Table 2 Differences in Endothelin-1, CBC, AST, ALT and Blood pressure between subjects

\begin{tabular}{|c|c|c|c|c|c|c|c|c|c|c|c|c|c|c|}
\hline & \multicolumn{5}{|c|}{$\begin{array}{l}\text { Preeclampsia } \\
\text { Cesarean section }(\mathrm{n}=20)\end{array}$} & \multicolumn{5}{|c|}{$\begin{array}{c}\text { Nonpreeclampsia } \\
\text { Cesarean section }(\mathrm{n}=25)\end{array}$} & \multicolumn{4}{|c|}{$\begin{array}{c}\text { Nonpreeclampsia } \\
\text { Vaginal Delivery }(\mathrm{n}=20)\end{array}$} \\
\hline & Before & Day 0 & Day 1 & Day 3 & Day 5 & Before & Day 0 & Day 1 & Day 3 & Day 5 & Before & Day 0 & Day 1 & Day 3 \\
\hline Endothelin-1 & 2.31 & $2.92 *$ & 2.43 & 2.62 & 1.89 & 1.79 & 2.12 & 2.19 & $2.53^{*}$ & 1.62 & 1.51 & $2.00 * *$ & 1.58 & 1.57 \\
\hline$(\mathrm{pg} / \mathrm{m} l)$ & $(0.75)$ & $(0.82)$ & $(0.70)$ & $(0.98)$ & $(0.56)$ & $(0.55)$ & $(0.59)$ & $(0.51)$ & $(0.84)$ & $(0.46)$ & $(0.37)$ & $(0.69)$ & $(0.58)$ & $(0.49)$ \\
\hline WBC & 7.83 & $12.20 * *$ & $14.30 * *$ & $9.62 *$ & 7.89 & 7.96 & $10.22 * * *$ & $12.25^{* *}$ & 8.26 & 5.93 & 7.78 & $10.35 * *$ & $11.25^{*}$ & $10.80^{*}$ \\
\hline$\left(* 10^{3} / \mu l\right)$ & $(1.90)$ & $(4.97)$ & $(4.80)$ & (3.28) & $(1.58)$ & $(2.50)$ & $(2.22)$ & $(3.45)$ & $(2.44)$ & $(1.20)$ & $(2.34)$ & $(1.74)$ & $(1.52)$ & $(1.52)$ \\
\hline $\mathrm{RBC}$ & 3.96 & 3.82 & 3.92 & 3.42 & 3.49 & 3.99 & 4.08 & 3.82 & 3.74 & 3.94 & 3.85 & 3.84 & 3.79 & 3.90 \\
\hline$\left(* 10^{6} / \mu l\right)$ & $(0.45)$ & $(0.57)$ & $(0.61)$ & $(0.62)$ & $(0.63)$ & $(0.38)$ & $(0.33)$ & $(0.25)$ & $(0.37)$ & $(0.51)$ & $(0.21)$ & $(0.24)$ & $(0.32)$ & $(0.22)$ \\
\hline Hemoglobin & 11.8 & 11.4 & $10.6^{*}$ & $10.0^{* *}$ & $10.2^{*}$ & 11.0 & 11.7 & 10.8 & 10.5 & 10.9 & 11.8 & 11.4 & 11.5 & 11.7 \\
\hline$(\mathrm{g} / \mathrm{d} l)$ & $(1.2)$ & (1.8) & (1.8) & (1.6) & (1.7) & (1.0) & $(0.9)$ & (1.6) & (1.1) & (1.4) & $(0.8)$ & (1.5) & (1.2) & (1.0) \\
\hline Hematocrit & 35.6 & 34.9 & 32.5 & $30.2 *$ & 31.2 & 33.4 & 34.5 & 33.1 & 32.5 & 34.6 & 35.4 & 34.7 & 35.0 & 36.2 \\
\hline$(\%)$ & $(4.1)$ & $(5.7)$ & $(6.7)$ & $(4.9)$ & (5.2) & (3.8) & (2.9) & $(4.8)$ & (3.3) & $(4.5)$ & $(2.2)$ & (3.5) & (3.5) & (3.7) \\
\hline Platelet & 219.7 & 194.4 & $184.4^{*}$ & 195.9 & $280.0^{*}$ & 264.7 & 267.5 & 259.4 & $307.2^{*}$ & $423.5^{*}$ & 264.0 & 235.7 & 240.2 & 254.0 \\
\hline$\left(* 10^{3} / \mu l\right)$ & $(73.7)$ & $(86.4)$ & $(70.0)$ & $(78.4)$ & $(80.0)$ & $(65.0)$ & (71.8) & $(68.7)$ & $(86.2)$ & (115.3) & $(15.0)$ & $(23.0)$ & $(24.8)$ & $(49.3)$ \\
\hline AST & 19.4 & 26.8 & 24.5 & $26.8^{*}$ & $27.3^{*}$ & 15.8 & $20.2^{*}$ & $21.1^{*}$ & $20.9^{*}$ & $19.7^{*}$ & 18.0 & 18.6 & 19.2 & 19.5 \\
\hline$(\mathrm{IU} / l)$ & (8.3) & (24.5) & (11.7) & $(14.0)$ & $(9.7)$ & $(4.7)$ & (3.3) & $(7.9)$ & $(8.9)$ & $(7.0)$ & (5.6) & $(7.1)$ & (8.0) & (7.9) \\
\hline ALT & 14.8 & 19.4 & 25.2 & 20.8 & 18.5 & 10.4 & 13.4 & 13.5 & $18.2 * * *$ & 17.6 & 11.3 & 11.8 & 12.0 & $17.2^{* * *}$ \\
\hline$(\mathrm{IU} / \mu l)$ & $(11.1)$ & (18.3) & $(34.2)$ & (15.8) & $(8.7)$ & $(8.3)$ & $(4.2)$ & $(5.4)$ & $(7.9)$ & $(8.1)$ & (5.4) & $(4.9)$ & $(4.2)$ & $(6.0)$ \\
\hline Systolic BP & 150.6 & 150.4 & 148.6 & 149.0 & $146.4^{*}$ & 110.8 & 110.0 & 108.8 & $106.0^{* * *}$ & 107.6 & 112.1 & 110.8 & 107.8 & 106.8 \\
\hline$(\mathrm{mmHg})$ & $(25.0)$ & (22.6) & $(20.2)$ & $(23.2)$ & $(22.0)$ & (18.6) & $(20.2)$ & $(18.0)$ & $(21.2)$ & $(17.2)$ & $(20.1)$ & (16.8) & $(12.6)$ & $(20.9)$ \\
\hline Diastolic BP & 96.4 & 97.4 & 95.3 & 96.2 & $92.6^{*}$ & 82.4 & 81.4 & 83.6 & $78.7^{* * *}$ & 80.4 & 81.6 & 80.5 & 78.8 & 78.4 \\
\hline (mmHg) & (21.8) & (20.6) & $(21.0)$ & $(24.1)$ & $(28.2)$ & (6.6) & $(11.2)$ & $(12.5)$ & (19.5) & $(16.4)$ & $(20.4)$ & $(16.8)$ & (18.0) & $(20.3)$ \\
\hline
\end{tabular}

$\mathrm{CBC}, \mathrm{WBC}, \mathrm{RBC}$ indicate complete blood count, white blood cell and red blood cell, respectively.

Average values are shown. Values in parenthesis indicate standard deviation of mean.

$*$, $* *$ and $* * *$ indicate $\mathrm{p}<0.05, \mathrm{p}<0.01$ and $\mathrm{p}<0.005$ vs values before delivery. 


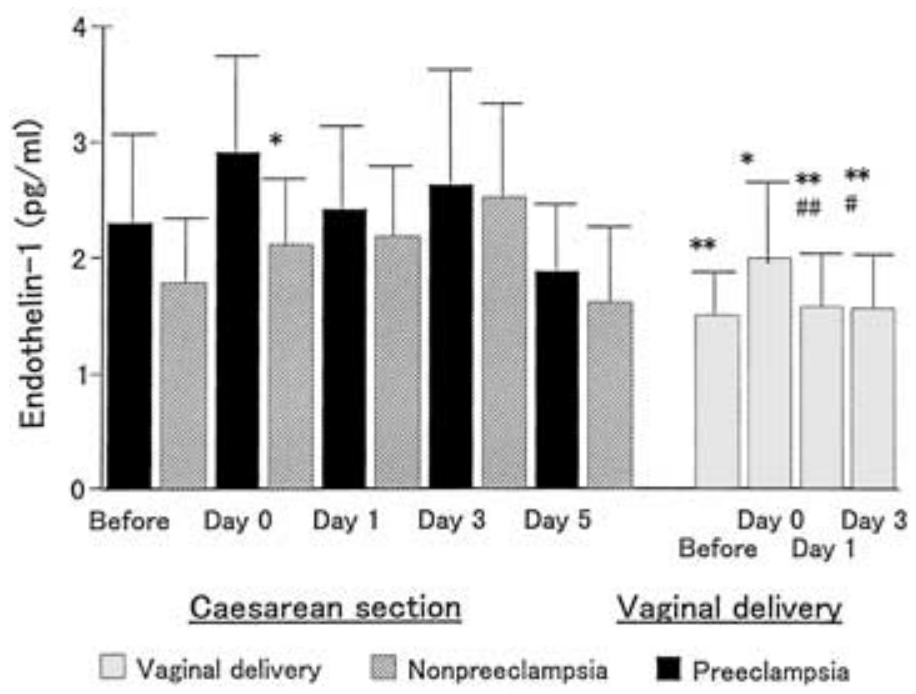

Fig. 1 Time course of plasma endothelin-1 levels after cesarean section. An explanation of each bar is provided in the graph. The vertical bars are the standard deviation. Asterisks $\left(^{*}\right)$ and

$\left({ }^{*}\right)$ indicate significance when compared with the value of preeclamptic women undergoing cesarean section at $\mathrm{p}<0.05$ and $\mathrm{p}<0.01$, respectively. Sharps $(\#)$ and (\# \#) indicate significance when compared with the value of nonpreeclamptic women undergoing cesarean section at $\mathrm{p}<0.05$ and $\mathrm{p}<0.01$, respectively. Each comparison is made between the values at same postpartal day.

observed between the women undergoing vaginal delivery and the preeclamptic and nonpreeclamptic women undergoing cesarean section when analyzed by two-way ANOVA $(\mathrm{p}<0.05)$. Plasma ET-1 concentration of the women undergoing vaginal delivery was lower compared with that of the preeclamptic women from before cesarean section to Day 3 after cesarean section $(\mathrm{p}<0.05)$.

Although the plasma ET-1 during pregnancy was not different between women without preeclampsia undergoing cesarean section and those undergoing vaginal delivery, the plasma ET-1 was significantly lower than that of non-preeclamptic women undergoing cesarean section on Day 1 and Day $3(\mathrm{p}<0.05)$.

\section{Platelet counts after cesarean section (Table}

\section{2, Fig. 2)}

(1) Cesarean section

Platelet counts were significantly different between the preeclamptic and non-preeclamptic women who underwent cesarean section when analyzed by two-way ANOVA $(\mathrm{p}<0.001)$. In the patients with preeclampsia, the mean platelet count was significantly lower than in the patients without preeclampsia before surgery $(219,700 \pm 73,700 / \mu l$ vs. $264,700 \pm$
$65,000 / \mu l$, in the women with preeclampsia and without preeclampsia, respectively, $(\mathrm{p}<0.05)$. Lower platelet counts in the women with preeclampsia continued after cesarean section, with the minimum value of the preeclamptic women $(189,400 \pm 70,000$ / $\mu l$ ) reached on Day 1 . The count then recovered to the preoperative value on Day 3. Then, the value on Day 5 increased from the preoperative value $(\mathrm{p}<$ $0.05)$.

In contrast, no decrease in the platelet counts after cesarean section was seen in the women without preeclampsia. From Day 5, the platelet count increased significantly from the preoperative value ( $p$ $<0.05)$.

Platelet counts in the non-preeclamptic women were significantly higher than those in the preeclamptic women during observation $(\mathrm{p}<0.05)$.

(2) Vaginal delivery

In the vaginal delivery group, platelet counts did not change on Day 0 and slightly increased on postpartum Day 3. However, the increase was not significant. Two-way ANOVA revealed a significant difference between the women who underwent vaginal delivery and the preeclamptic women who underwent cesarean sections $(\mathrm{p}<0.005)$. Platelet counts of 


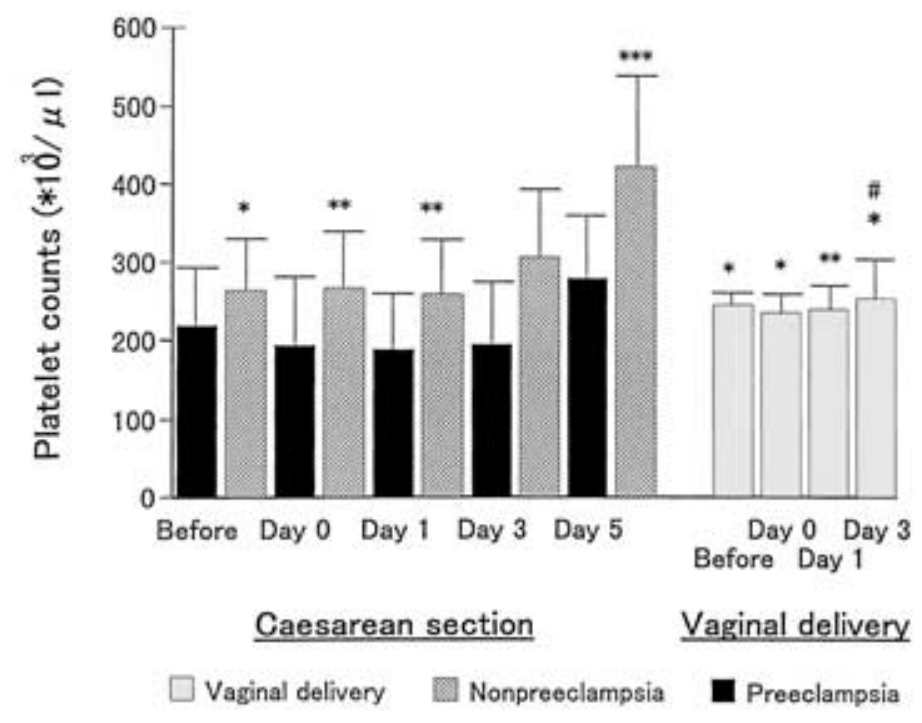

Fig. 2 Time course of platelet counts after cesarean section. See the legend of the fig. 1 for explanations of bars, asterisks $(*)$ and $\left({ }^{*}\right)$.

the women undergoing vaginal delivery were significantly higher compared with those of the preeclamptic women from before delivery to postpartum Day $3(\mathrm{p}<0.05)$. In contrast, no difference from non-preeclamptic women undergoing cesarean sections was found.

4. WBC, RBC, hemoglobin and hematocrit after cesarean section (Table 2)

(1) Cesarean section

WBC increased immediately after delivery in both groups. Hemoglobin and hematocrit decreased after cesarean section in the preeclamptic women $(\mathrm{p}<$ 0.05). No significant differences in WBC, hemoglobin or hematocrit were observed among the preeclamptic and non-preeclamptic women who underwent cesarean section.

(2) Vaginal delivery

No significant differences were observed between the women who underwent vaginal delivery and the women who underwent cesarean sections by twoway ANOVA.

\section{AST and ALT after cesarean section. (Table 2)}

(1) Cesarean section

Plasma levels of AST in the preeclamptic women significantly increased by Day $3(\mathrm{p}<0.05)$. The pregnant women without preeclampsia demonstrated the same tendency as the preeclamptic women $(\mathrm{p}<0.05)$.

In contrast, ALT in the women with preeclampsia tended to increase after cesarean section, but the increases were not significant compared with the preoperative value.

Both the AST and ALT of the pregnant women with preeclampsia tended to be high, compared with those of the women without preeclampsia, but no significant differences were observed between the groups by two-way ANOVA.

(2) Vaginal delivery

AST in the women undergoing vaginal delivery did not change according to the time course postpartum. ALT increased on Day 3 from the level before delivery $(p<0.005)$. No significant differences were observed in the AST and ALT of the pregnant women who underwent vaginal delivery and those who underwent caesarean section.

\section{Blood pressure}

(1) Cesarean section

Systolic and diastolic blood pressures were high by Day 3 in patients with preeclampsia and a significant decrease from the preoperative value was found on Day $5(\mathrm{p}<0.05)$.

In the women without preeclampsia, both systolic and diastolic blood pressures decreased by Day 3 after caesarean section $(\mathrm{p}<0.001)$. 


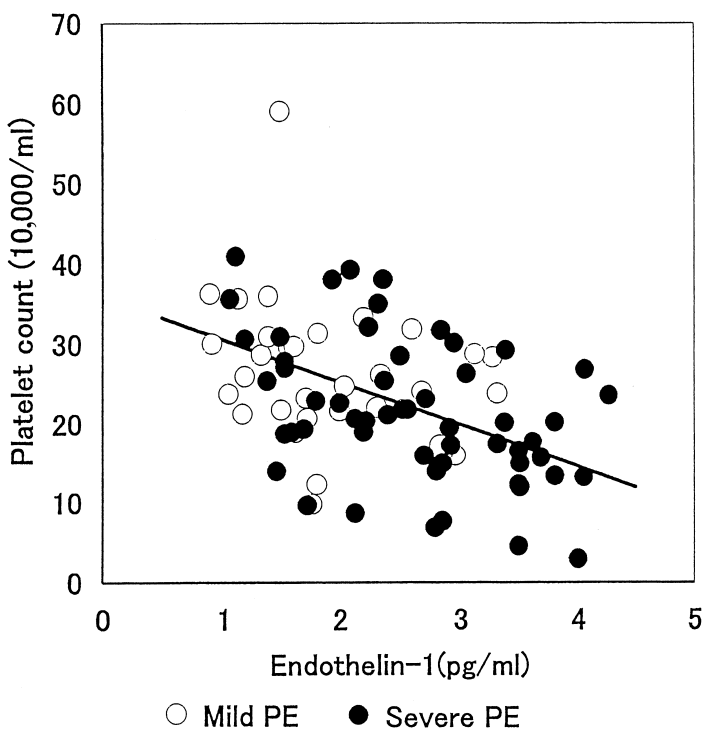

Fig. 3 Relationship between endothelin-1 and platelet counts after cesarean section in patients with preeclampsia. A linear correlation was seen between the two variables ([platelet count $]=360,000-5,350 \times$ [endothelin- 1$])(\mathrm{r}=$ $0.46, \mathrm{p}<0.001, \quad[\mathrm{n}=96])$

A significant difference in blood pressure was observed between preeclampsia and non-preeclampsia $(\mathrm{p}<0.001)$.

(2) Vaginal delivery

Blood pressure did not change significantly with time postpartum. The pressures were lower than in the women with preeclampsia $(p<0.001)$, but were not different from those in the non-peeclamptic women who underwent cesarean section.

\section{Relationship between plasma ET-1 and other} variables

(1) Relationship between platelet counts and ET-1

(Fig. 3)

A negative correlation was observed between preoperative and postoperative plasma ET-1 concentrations and platelet counts in the women with preeclampsia who underwent cesarean section (preoperative: $\mathrm{r}=0.36$; postoperative: $\mathrm{r}=0.46$; $\mathrm{p}<0.05$ ). Preoperative ET-1 did not significantly correlate with platelet counts. In contrast, no correlation was observed between either the pre-or postoperative values for the non-preeclamptic women who underwent cesarean section and vaginal delivery.

(2) Relationship between ET-1 and blood pres- sure.

A positive correlation was identified between preoperative systolic blood pressure and ET-1 levels in the preeclamptic women who underwent cesarean section $(r=0.52, p<0.001)$. A similar trend was seen after surgery but there was no significant relationship. Diastolic blood pressures did not show any correlation with ET-1 levels. No correlation was observed between the blood pressure and ET-1 of the nonpreeclamptic women who underwent cesarean sections and vaginal delivery.

(3) Relationship between ET-1 and ALT, AST and complete blood cell counts except platelet counts.

No relationship was observed between ET-1 and ALT, AST and complete blood cell counts, except platelet counts.

\section{Discussion}

In pregnant women with preeclampsia, the plasma ET-1 level was high before surgery according to the severity of hypertension and decrease in platelet count. This was consistent with the results of previous studies ${ }^{9-11}$. A novel finding was made regarding women with preeclampsia: plasma ET-1 concentration rose above the preoperative value on Day 0 and then tended to fall afterwards. But the decrease from Day 0 was not significant by Day 5. Furthermore, the postoperative ET-1 level was found to be closely associated with a decrease in platelet count. This was not observed in the non-preeclamptic women who underwent cesarean section and vaginal delivery.

In patients with preeclampsia, it is highly likely that vascular endothelial injury, resulting in chronic intravascular coagulation, is already present. This vascular endothelial condition leads to the enhancement of ET-1 production ${ }^{10}$. Therefore, it is possible that a cesarean section performed in such a state causes additional stimulation of ET-1 production in the early postoperative period. Surgical stress stimulates ET-1 production immediately after surgery ${ }^{1-3}$. Nelson et al. showed enhanced ET-1 production immediately after surgery, with levels remaining high until 3 hours after surgery and then falling on 
postoperative Day one $e^{2}$ This is thought to reflect respiratory and circulatory stress, along with hemodynamic changes, and hypoxia and ischemia at the tissue level as a result of surgery. Since we did not measure ET-1 until 6 hours after cesarean section, we could not accurately compare the time course of ET-1 levels after surgical procedures between our preeclamptic patients and Nelson's subjects. However, considering the fact that the plasma half-life of ET-1 is short (approximately 3.5 minutes) ${ }^{18}$, the high concentration of ET-1 found in preeclamptic women on Day 0 (6 12 hours) after cesarean section suggests that enhanced production of ET-1 may be sustained by this time. Furthermore, high concentrations of ET-1 were observed until postoperative Day 3. In contrast, it has been reported that ET-1 concentrations increased on day of vaginal delivery but fell afterwards ${ }^{19}$, as observed in the present subjects undergoing vaginal delivery. Therefore, the findings of high concentrations of plasma ET-1 by Day 3 in women without preeclampsia and peak concentration in preeclamptic women on Day 0 followed by high levels were clearly different from those found after vaginal delivery.

Wound healing after surgical stress can be divided into three stages which occur over time: inflammatory stage, tissue formation and tissue remodeling. The period up to postoperative days $3 \sim 4$ corresponds to the stages from inflammation to tissue formation $^{20}$. At this time, physiological substances which enhance ET-1 production, such as transforming growth factor- $\alpha$, epidermal growth factor and catecholamine are locally released ${ }^{1}$. Therefore, one could assume that the rise in plasma ET-1 levels in the non-preeclamptic pregnant women at this time reflect at least an increase in ET-1 production due to stimulation local to the wound site after cesarean section $^{7.20}$. The mechanism by which ET-1 production is stimulated in the postoperative period may differe between women with and without preeclampsia. We need further investigations that include various profiles of surgical stress that stimulate production of endothelin-1 as well as other vasoactive substances during the process of wound healing.

As was observed in the time course of ET-1 after cesarean section, AST and ALT levels have been re- ported to increase after surgical procedures in nonpreeclamptic women ${ }^{21}$. This is true on the present results for AST and ALT in preeclamptic and nonpreeclamptic women after cesarean section. Tissue trauma of uterine smooth muscle due to cesarean section is assumed to cause the release of more ALT and AST from injured cells into the circulation ${ }^{22}$. AST and ALT after cesarean section were not different between our preeclamptic and nonpreeclamptic women.

ET-1 has binding sites at both vascular and nonvascular sites distributed throughout the cardiac tissue, renal tissue, adrenal glands, central nervous system, lungs and gastrointestinal tract ${ }^{23,24}$. High concentrations of plasma ET-1 after cesarean section may result not only from increased synthesis and release but also from decreased extraction and degradation of ET-1. As for the mechanism of the high concentration of ET-1 after cesarean section, there are no reports at present. Therefore, further study is necessary to focus on the regulation of ET-1 after cesarean section from the viewpoint of change in these binding sites between women with and without preeclampsia.

It has been thought that ET-1 is related to the pathogenesis of HELLP syndrome, as well as preeclampsia, by its regulatory functions on vascular tone and potent vasoconstrictive effects ${ }^{1-3}$. However, the main organ responsible for high plasma levels of ET-1 in women with HELLP syndrome and preeclamptic pregnant women has not been clarified. Häkkinnen et al suggested ET-1 is mainly released from the placenta during delivery, because of much higher concentrations of ET-1 in the retroplacental blood than in maternal plasma and cord blood ${ }^{19}$. If so, from immediately after cesarean section, the responsible organ, where vascular endothelial cells mainly release ET-1, may change from the placenta to injured tissues due to cesarean section. Or extraction or degradation of ET-1 may increase in other tissues. We should await future studies to clarify this.

ET-1 increases platelet aggregation evoked by adenosine diphosphate ${ }^{12}$ and causes platelet activa$\operatorname{tion}^{13}$. Platelet consumption and thrombus formation have been reported after intravenous injection of ET-1 in in-vivo animal studies ${ }^{14,25}$. Furthermore, 
preeclampsia is known to be related with platelet activation and intravascular platelet consumption ${ }^{26-28}$. Thus, it may be reasonable to consider that increased ET-1 is closely associated with intravascular platelet consumption in preeclamptic women even after cesarean section. According to Shibai, 31\% of HELLP syndrome cases occur postpartum ${ }^{29}$. However, there is no literature concerning evidence of whether cesarean section for preeclampsia is a predisposing factor for postpartal thrombocytopenia and HELLP syndrome to our knowledge. Also, our results did not produce any clear evidence to clarify whether surgical invasion associated with cesarean section itself changes platelet function and causes the onset of thrombocytopenia. However, we did find a high plasma concentration of ET-1 levels in the early postoperateive days and a relationship between increased ET-1 levels and decreased platelet counts in women with preeclampsia. It is clinically relevant to protect thrombocythopenia after cesarean section. Future investigations should aim to clarify the possibility that cesarean section in patients with preeclampsia may lead to postpartal thrombocytopenia.

\section{References}

1. Hirata $\mathrm{Y}$, Itoh $\mathrm{K}$, Ando $\mathrm{K}$, Endo $\mathrm{M}$, Marumo $\mathrm{F}$ : Plasma endothelin levels during surgery. N Engl J Med 1989; 321: 1689.

2. Nelson JB, Opennorth TJ, Fleisher LA, Frank SM: Perioperative plasma endothelin-1 and big endothelin-1 concentrations in elderly patients undergoing major surgical procedures. Anesth Analg 1999; 88: 898-903.

3. Itoh K, Gosek N, Endo M: Intraoperative hemorrhage affects endothlin-1 concentrations. Am J Gastroenterol 1991; 86: 118-119.

4. Gensini GF, Conti AA, Prisco D: Endothlins: an overview of recent achievements. Ann Ital Med Int 1998; 13: 88-92

5. Noiri E, Hu Y, Bahou WF, Keese CR, Giaever I, Goligordky MS: Permissive role of nitric oxide in endothlin-induced migration of endothelial cells. J Biol Chem 1997; 272: 1747-1752.

6. Komoro I, Kurihara T, Takaku F, Yazaki Y: Endothelin stimulates c-fos and c-myc expression and proliferation of vascular smooth muscle cells. FEBS letter 1988; 238: 249-252.

7. Takuwa Y, Yanagizawa M, Takuma N, Masaki T: Endothelin, its diverse biological activities and mechanism of action. Prog Growth factor Res 1989;
$1: 195^{-206}$

8. Gologorsky MS, Budzikowsky AS, Tsukahara H, Noiri E: Co-operation between endothelin and nitric oxide in prompting endothelial cell migration and angiogenesis. Clin Exp Pharmcol \& Physiol 1999;26: 269-271.

9. Nova A, Shibai BM, Barton JR, Mercer BM, Mitchel MD: Maternal plasma levels of endothelin are increased in preeclampsia. Am J Obstet Gynecol 1991; 165: 724-727.

10. Ferreria-de-Amedia J, Amenta F, Cardoso F, Po' lonia J: Association of circulating endothelin and noradrenaline with increased calcium-channel binding sites in the placental bed in pre-eclampsia. Br J Obstet Gynecol 1998; 105: 1104-1112.

11. Srefanie B, Sutterlin M, Steck T: Plasma endothelin and big endothelin levels in women with severe preeclapsia or H ELLP-syndrome. Arch Gynecol Obstet 1999; 262: 113-119.

12. Kohler R, Urano T, Malyszko J, Takada Y, Takada A: In vitro effect of endothelin-1 on collagen and ADP-induced aggregation in human whole blood and platelet rich plasma. Thromb Res 1994; 77: 69-78.

13. Halim A, Kanayama N, Marady ELE, Maehara K, Hirano M, Kobayashi T, Terao T: Endothelin-1 evoked an increase and oscillation in cytosolic calcium concentration in adherent single human platelets and increased GMP-140 (P-selectinin) in platelet suspension. Thromb Res 1995; 80: 105-112.

14. Halim A, Kanayama N, Maradney EEL, Maehara K, Terao T: Coagulation in vivo microcirculation and in vitro caused by endothelin-1. Tronmb Res 1993; 72: 203-209.

15. Neiger R, Contag SA, Coustan DR: The resolution of preeclampsia related thrombocytopenia. Obstet Gynecol 1991; 77: 692-695.

16. Chandran R, Serra-Serra V, Redman CWG: Spontaneous resolution of preeclamsia-related thrombocytopenia. Br J Obstet Gynecol 1992; 99: 887-890.

17. Futaguchi $\mathrm{Y}$, Ishiguro $\mathrm{Y}$, Watanabe S, Yonezawa M, Tabata N:Basic research on the measurement of plasma endothelin-1 by Octadecylsilyl silica (ODS) extraction (Author's translation). Kakuigaku Gijyutu 1993; 14: 9-13 (in Japanese).

18. Vierhapper H, Wagner O, Nowotny P, Waldhaus W: Effect of endothelin-1 in man. Circulation 1990;81: 1415-1418.

19. Häkkinnen LM, Voulteenaho OJ, Leppluoto JP, Laatikainen TJ: Endothelin in maternal and umbilical cord blood in spontaneous labor and at elective cesarean delivery. Obstet Gynecol 1992; 80: 72-75.

20. Singer AJ, Clark RAF: Cutaneous wound healing. New Engl J Med 1999; 10: 738-745.

21. David AL, Kotecha M, Girling JC: Factors influencing postnatal liver function test. Br J Obstet Gynecol 2000; 107: 1421-1426.

22. Person DA, Judge RD: Effect of operation on serum transaminase levels. Arch Surg 1958; 77: 892-897.

23. Gerebes AL, Mథller S, Gülberg V, Henriksen H; Endothelin-1 and -3 plasma concentrations in patients with cirrhosis: role of splanchnic and renal pas- 
sage and liver function. Hepatology 1995; 21: 735-739.

24. Randal MD: Vascular activities of the endothelins. Pharmac Ther 1991; 50: 73-93.

25. Helset E, Lindal S, Olsen R, Mykleburn P, Jorgensen L: Endothelin-1 caused sequential trapping of platelets and neutrophiles in the pulmonary microcirculation in rats. Am J Obstet Physiol 1996; 271 (4 Pt 1): L 538-546.

26. Saleh AA, Bottoms SF, Farag AM, Dombrowski MP, Welch RA, Norman G, Mammer EF: Markers for endothelical injury, clotting and platelet activation in preeclampsia. Arch Gynecol Obstet 1992: 251: 105110.

27. Perry KG, Martin JN: Abnormal hemostasis and co- agulopathy in preeclampsia and eclampsia. Clin $\mathrm{Ob}$ stet Gynecol 1992; 35: 338-350.

28. Remuzzi G, Ruggenenti P: Prevention and treatment of pregnancy-associated hypertension: what we learned in the last 10 years? Am J Obstet Gynecol 1991; 18: 285-305.

29. Shibai BM: The HELLP syndrome (hemolysis, elevated liver enzymes and low platelets): much ado about nothing? Am J Obstet Gynecol 1990; 162: 311316.

(Received, May 12, 2003)

(Accepted, July 3, 2003) 